REGARDS

SUR L'ECONOMIE ALLEMANDE

BULLETIN ECONOMIQUE DU CRAC

\section{Regards sur l'économie allemande}

Bulletin économique du CIRAC

$105 \mid 2012$

Varia

\title{
Le coût économique du chômage est en baisse en
} RFA

\author{
Hans-Uwe Bach et Eugen Spitznagel
}

Traducteur : Isabelle Bourgeois

\section{OpenEdition}

Journals

Édition électronique

URL : http://journals.openedition.org/rea/4421

DOI : $10.4000 /$ rea. 4421

ISBN : 978-2-8218-1420-2

ISSN : 1965-0787

Éditeur

CIRAC

Édition imprimée

Date de publication : 11 juillet 2012

Pagination : 27-36

ISSN : 1156-8992

\section{Référence électronique}

Hans-Uwe Bach et Eugen Spitznagel, "Le coût économique du chômage est en baisse en RFA »,

Regards sur l'économie allemande [En ligne], 105 I juillet 2012, mis en ligne le 01 juillet 2014, consulté le 21 avril 2019. URL : http://journals.openedition.org/rea/4421 ; DOI : 10.4000/rea.4421 


\section{Le coût économique du chômage est en baisse en RFA}

\author{
Hans-Uwe Bach, Eugen Spitznagel
}

Le chômage pèse sur les finances des ménages, mais il grève aussi les finances publiques. En situation de sous-emploi, qu'il s'agisse de chômage visible ou caché, les salariés ne perçoivent qu'une rémunération faible, voire nulle. Il en résulte des pertes à l'échelle macroéconomique : l'Etat est amené à augmenter ses dépenses alors même que ses recettes se réduisent. Ces coûts supplémentaires pèsent sur l'ensemble des comptes publics; les budgets de l'Etat fédéral, des Länder, des communes et des organismes de protection sociale sont touchés soit directement, soit indirectement en raison de leur interdépendance. Ainsi, en 2011, en comparaison avec une situation hypothétique où il n'y aurait eu aucun chômage, le coût budgétaire induit par le chômage s'élevait à quelque 56 milliards $€$ en Allemagne.

L'impact du chômage sur les ménages et l'ensemble des budgets publics est fonction notamment des prestations allouées. Dans ce domaine, d'importants changements ont eu lieu ces dernières années: le système de protection sociale pour les demandeurs d'emploi a été profondément remanié en 2003-2005. Ces réformes (" réformes Hartz ») avaient pour objectif premier d'accroître l'incitation à la reprise d'un emploi. Les économies réalisées devaient ouvrir des marges de manœuvre permettant de réduire les cotisations d'assurance chômage. Enfin, les réformes devaient clarifier les compétences budgétaires respectives en ce qui concerne le financement des prestations d'assurance d'un côté, celui des prestations supportées par l'ensemble de la société, de l'autre, ceci entre autres pour éviter que ne se reproduisent les problèmes de pilotage observés par le passé (" erreurs d'aiguillage » budgétaires).

L'Agence fédérale pour l'emploi estime que ces réformes sont un succès à deux égards: elles ont permis d'une part de réduire les coûts supportés par l'assurance chômage et, d'autre part, de séparer les prestations d'assurance des prestations d'assistance en asseyant leur financement sur des bases indépendantes l'une de l'autre, c'est-à-dire sur les cotisations dans le premier cas, sur l'impôt dans le deuxième. Reste à en évaluer le coût social global...

\section{Coût budgétaire global généré par le chômage}

Après avoir atteint un niveau record de plus de 6 millions de personnes en 2006, le sous-emploi dans son ensemble (pour la définition du sous-emploi, voir encadré infra) s'est réduit de quelque 2,2 millions, pour se situer à près de 4,3 millions de personnes en 2011. Durant la récession de 2009, ce chiffre avait connu une légère hausse passagère (Fuchs et al., 2012).

Comme toujours dans de tels cas, les ressources économiques restent pour une très large part inexploitées, la création de valeur restant inférieure au potentiel productif. Si ce 'manque à gagner' reflète le coût macroéconomique du sous-emploi, il n'est cela dit guère possible de l'évaluer avec précision (Horn/ Tober, 2007 et Bach/Spitznagel, 2008). Et ce notamment en raison de la difficulté à estimer la productivité potentielle de la réserve latente. Dès lors, dans l'analyse des coûts ci-après, nous nous limiterons à estimer l'ensemble des coûts budgétaires induits par les chômeurs inscrits, la réserve latente n'étant ici pas prise en compte.

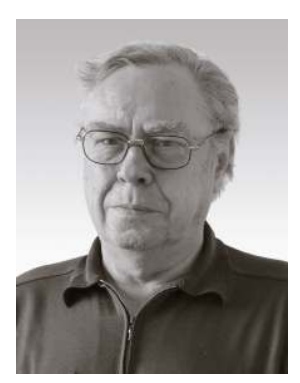

Hans-Uwe Bach, chercheur émérite, Groupe de recherche Temps de travail et marché du travail de l'IAB (Nuremberg)

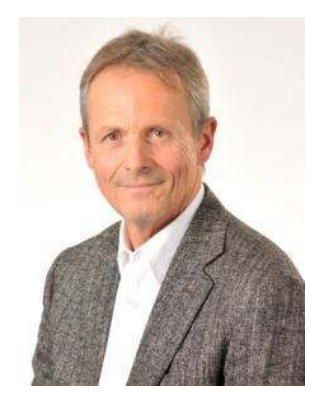

Eugen Spitznagel, Directeur du Groupe de recherche Marché du travail et métiers de l'IAB (Nuremberg) 
L'IAB et l'Agence fédérale pour l'emploi ne retiennent pas la même définition du sous-emploi (Unterbeschäftigung) que l'INSEE et le BIT.

Pour ces derniers, le sous-emploi « comprend les personnes actives occupées au sens du BIT qui remplissent l'une des conditions suivantes:

- elles travaillent à temps partiel, souhaitent travailler davantage pendant la période de référence utilisée pour définir l'emploi, et sont disponibles pour le faire, qu'elles recherchent activement un emploi ou non ;

- elles travaillent à temps partiel (et sont dans une situation autre que celle décrite ci-dessus) ou à temps complet, mais ont travaillé moins que d'habitude pendant une semaine de référence en raison de chômage partiel, ralentissement des affaires, réduction saisonnière d'activité ou mauvais temps ».

Pour l'IAB et l'Agence fédérale pour l'emploi en revanche, le sous-emploi rassemble les chômeurs inscrits, les personnes participant à des mesures d'insertion professionnelle ou sociale (tant que celles-ci ne travaillent pas) et les demandeurs d'emploi non inscrits à l'Agence fédérale pour l'emploi $(\mathrm{SH})$.

Réserve latente un vaste ensemble

Le sous-emploi génère des coûts

56 milliards $€$ au total en 2011 pour les chômeurs inscrits

Dépenses : 31 milliards $€$
Les chômeurs inscrits à l'Agence fédérale pour l'emploi constituent l'essentiel du sous-emploi (données administratives), à quoi s'ajoute le chômage dit caché, sous ses différentes formes. II convient en effet de tenir compte de cette « réserve latente »(3,8 millions de personnes en juin 2012) à la fois sous l'angle de la politique active pour l'emploi, celui de la politique sociale et de la politique budgétaire. On distingue deux catégories: d'une part la réserve latente " en mesures » qui comprend les personnes prenant part à des mesures d'insertion/réinsertion professionnelle ou des mesures sociales de nature diverse (formation professionnelle continue, mesures de développement des compétences, réglementations s'apparentant aux mesures de préretraite...). La réserve latente stricto sensu, d'autre part, comprend les personnes qui, si le marché du travail offrait un contexte plus favorable, seraient plus activement à la recherche d'un emploi, mais qui ne sont ni inscrites au chômage, ni bénéficiaires d'aucune mesure d'insertion.

En principe, tous les chômeurs et, par-delà, toutes les personnes constituant la réserve latente peuvent contribuer à l'activité macroéconomique et percevoir un salaire. Dans ce cas théorique, aucune dépense d'assurance chômage ou d'assistance n'est à prévoir, chacun contribuant par ailleurs à l'impôt et aux cotisations sociales. Dans la réalité néanmoins, il existe toujours une certaine part de chômage conjoncturel, saisonnier, frictionnel ou structurel, générant des coûts qu'il s'agit de contenir le plus possible.

Les données présentées ci-dessous se fondent sur des calculs types effectués sur la base d'hypothèses simplifiées. Ils partent notamment du principe que les chômeurs pourraient être recrutés à des conditions (salaire, temps de travail) identiques à celles de leur dernier poste ; le moins-perçu est alors estimé en fonction de ce salaire théorique. Quant aux dépenses réalisées pour les allocations chômage et pour l'assurance sociale des chômeurs, elles sont issues pour l'essentiel des données de l'Agence fédérale pour l'emploi.

\section{Dépenses et moins-perçus de l'année 2011}

En 2011, l'ensemble des coûts budgétaires induits par les chômeurs inscrits se montait à 56,4 milliards $€$ en Allemagne, soit plus de 31 milliards $€$ de moins qu'en 2005 (voir tableau 1).

Les dépenses consacrées aux prestations se taillaient la part du lion avec 31 milliards $€$, soit $55 \%$ du total des coûts (contre $53 \%$ en 2005). Elles se composent de l'allocation chômage (Arbeitslosengeld I, ALG I) et de l'aide forfaitaire s'appliquant aux demandeurs d'emploi ne bénéficiant plus de l'indemnité de chômage (Arbeitslosengeld II, ALG II) ; elles incluent dans les deux cas les cotisations sociales. L'aide versée au titre de l'ALG II (" Hartz IV ») est un forfait de base destiné à garantir un minimum vital ; s'y ajoutent, le cas échéant, des aides pour les frais de logement et de chauffage ainsi que pour divers besoins supplémentaires, versées au foyer du chômeur. Font également partie des dépenses d'éventuels montants compensatoires pour les bénéficiaires de l'ALG I dont l'allocation est inférieure à l'aide forfaitaire de base définie au chapitre II du 
Code social allemand (SGB II). Jusqu'en 2010 par ailleurs, un complément était temporairement accordé lors du passage de l'ALG I vers l'ALG II (§ 24, SGB II). Enfin, les dépenses comprennent également l'allocation sociale (Sozialgeld) versée aux personnes en incapacité de travailler appartenant à la communauté de besoins des chômeurs (conjoint, enfants...) ; car si ces derniers n'étaient pas au chômage, cette allocation ne serait pas versée aux personnes vivant sous leur toit.

Tableau 1. Coût budgétaire global du chômage en RFA 2001-2011

\begin{tabular}{|c|c|c|c|c|c|c|c|c|c|c|c|}
\hline & 2001 & 2002 & 2003 & 2004 & 2005 & 2006 & 2007 & 2008 & 2009 & 2010 & 2011 \\
\hline \multicolumn{12}{|c|}{ en milliers } \\
\hline Chômeurs inscrits ${ }^{\text {a) }}$ & 4219 & 4535 & 4830 & 4812 & 4861 & 4487 & 3776 & 3268 & 3423 & 3245 & 2976 \\
\hline \multicolumn{12}{|c|}{ en milliers $€$ par an } \\
\hline Coût par chômeur & 18,2 & 18,4 & 18,9 & 19,2 & 18,0 & 18,3 & 17,8 & 17,1 & 17,5 & 18,5 & 18,9 \\
\hline $\begin{array}{l}\text { Transferts par chômeur } \\
\text { b) }\end{array}$ & 6,6 & 6,7 & 6,8 & 6,9 & 6,5 & 6,7 & 6,8 & 6,7 & 7,2 & 7,5 & 7,6 \\
\hline \multicolumn{12}{|c|}{ en milliards $€$} \\
\hline $\begin{array}{l}\text { Coût budgétaire global } \\
\text { dont }\end{array}$ & 76,7 & 83,7 & 91,5 & 92,2 & 87,7 & 82,2 & 67,2 & 55,9 & 59,8 & 60,2 & 56,4 \\
\hline prestat. assurance & 22,2 & 24,1 & 25,1 & 24,7 & 22,2 & 17,6 & 12,3 & 9,0 & 13,8 & 14,0 & 12,1 \\
\hline prestat. sociales & 18,2 & 20,0 & 21,5 & 23,3 & 24,6 & 25,7 & 22,7 & 20,5 & 20,6 & 20,2 & 19,1 \\
\hline déficit encours fiscal & 14,8 & 16,1 & 17,7 & 17,3 & 16,2 & 15,0 & 12,1 & 9,8 & 10,0 & 10,3 & 9,5 \\
\hline déficit cotisations & 21,5 & 23,5 & 27,1 & 26,9 & 24,7 & 23,8 & 20,1 & 16,5 & 15,4 & 15,7 & 15,6 \\
\hline \multicolumn{12}{|c|}{ Plus indemnité de fin de droits (Aussteurungsbeitrag) / contribution d'insertion (Eingliederungsbeitrag) } \\
\hline Surcroît dépenses BA / su & plus rec & es Bund & & & 3,7 & 2,5 & 1,4 & 3,3 & 3,9 & 4,4 & 4,0 \\
\hline \multicolumn{12}{|c|}{ Parts respectives en \% } \\
\hline $\begin{array}{l}\text { Coût budgétaire global } \\
\text { dont }\end{array}$ & 100,0 & 100,0 & 100,0 & 100,0 & 100,0 & 100,0 & 100,0 & 100,0 & 100,0 & 100,0 & 100,0 \\
\hline prestat. assurance & 28,9 & 28,8 & 27,5 & 26,8 & 25,3 & 21,4 & 18,2 & 16,2 & 23,0 & 23,2 & 21,6 \\
\hline prestat. sociales & 23,7 & 23,9 & 23,5 & 25,3 & 28,0 & 31,3 & 33,8 & 36,8 & 34,5 & 33,5 & 33,9 \\
\hline déficit encours fiscal & 19,3 & 19,3 & 19,4 & 18,8 & 18,5 & 18,3 & 18,0 & 17,5 & 16,7 & 17,1 & 16,8 \\
\hline déficit cotisations & 28,0 & 28,1 & 29,7 & 29,2 & 28,2 & 29,0 & 30,0 & 29,5 & 25,8 & 26,1 & 27,7 \\
\hline
\end{tabular}

Source des données : calculs de l'IAB. a) De 2001 à 2004, cette catégorie inclut également le nombre estimé de bénéficiaires de l'aide sociale (ancien régime) capables de travailler et qui, après les réformes entrées en vigueur en 2005, auraient été comptabilisés chômeurs (voir à ce propos IAB-Kurzbericht, $\left.n^{\circ} 14 / 2008\right)$. b) changement de types de prestations en 2005.

Tableau 2. Répartition du coût du chômage sur les budgets publics 2001-2011 *)

\begin{tabular}{|c|c|c|c|c|c|c|c|c|c|c|c|}
\hline & 2001 & 2002 & 2003 & 2004 & 2005 & 2006 & 2007 & 2008 & 2009 & 2010 & 2011 \\
\hline \multicolumn{12}{|c|}{ en milliards $€$} \\
\hline Agence f. pour l'emploi & 27,6 & 30,0 & 31,5 & 31,1 & 32,1 & 25,9 & 16,7 & 14,3 & 19,4 & 20,2 & 17,8 \\
\hline Bund & 19,0 & 21,2 & 23,5 & 25,3 & 22,4 & 23,8 & 21,2 & 16,4 & 15,7 & 14,7 & 13,9 \\
\hline Länder & 6,7 & 7,3 & 7,9 & 7,7 & 6,7 & 6,2 & 5,0 & 4,0 & 4,2 & 4,3 & 3,9 \\
\hline Communes & 7,2 & 7,6 & 7,8 & 7,6 & 8,0 & 8,3 & 7,2 & 6,6 & 6,9 & 7,0 & 6,7 \\
\hline Assurance maladie & 6,0 & 6,7 & 8,1 & 8,2 & 7,0 & 6,8 & 6,0 & 4,9 & 4,6 & 4,7 & 4,3 \\
\hline Assurance retraite & 9,3 & 10,0 & 11,7 & 11,2 & 10,7 & 10,4 & 10,5 & 8,9 & 8,4 & 8,5 & 9,1 \\
\hline Assurance dépendance & 0,8 & 0,9 & 1,0 & 1,0 & 0,8 & 0,8 & 0,7 & 0,7 & 0,6 & 0,7 & 0,6 \\
\hline \multicolumn{12}{|c|}{ Parts respectives en \% } \\
\hline Agence f. pour l'emploi & 36,0 & 35,8 & 34,4 & 33,8 & 36,6 & 31,5 & 24,9 & 25,6 & 32,5 & 33,6 & 31,6 \\
\hline Bund & 24,8 & 25,3 & 25,7 & 27,4 & 25,5 & 29,0 & 31,6 & 29,4 & 26,2 & 24,5 & 24,7 \\
\hline Länder & 8,8 & 8,7 & 8,7 & 8,4 & 7,6 & 7,5 & 7,4 & 7,2 & 7,0 & 7,1 & 7,0 \\
\hline Communes & 9,4 & 9,1 & 8,5 & 8,2 & 9,2 & 10,1 & 10,7 & 11,8 & 11,5 & 11,6 & 12,0 \\
\hline Assurance maladie & 7,8 & 8,0 & 8,8 & 8,9 & 7,9 & 8,3 & 8,9 & 8,7 & 7,7 & 7,9 & 7,5 \\
\hline Assurance retraite & 12,1 & 11,9 & 12,8 & 12,2 & 12,2 & 12,6 & 15,6 & 15,9 & 14,0 & 14,1 & 16,1 \\
\hline Assurance dépendance & 1,1 & 1,1 & 1,1 & 1,1 & 0,9 & 1,0 & 1,0 & 1,3 & 1,1 & 1,1 & 1,1 \\
\hline
\end{tabular}

Source des données : calculs de l'IAB. *) Le nombre de chômeurs considéré inclut, sur la période 2001-2004, le nombre estimé de bénéficiaires de l'aide sociale (ancien régime) capables de travailler et qui, après les réformes entrées en vigueur en 2005, auraient été comptabilisés chômeurs.

Pour les budgets publics, cette situation se solde par un déficit de recettes estimé à 25 milliards $€$, soit $45 \%$ du total des coûts budgétaires liés au chômage Déficit de recettes : (contre $47 \%$ en 2005). II résulte essentiellement d'une baisse des recettes de 25 milliards $€$ cotisations sociales et, dans une moindre mesure, de celle de l'encours fiscal toujours en comparaison respective avec une situation de plein emploi absolu. 
Les caisses d'assurance sociale sont les plus touchées

9,5 milliards $€$ d'impôts non perçus

Coûts variables selon les chômeurs

Les « réformes Hartz » on fait baisser les coûts
Les caisses d'assurance sociale engrangent moins de recettes, car les cotisations dues par les chômeurs allocataires sont plus faibles que celles dues sur le salaire. Avec près de 9,1 milliards $€$, c'est l'assurance retraite qui enregistre le plus important déficit en 2011 ; pour l'assurance maladie, il s'élève à 4,3 milliards $€$, et tout de même à 600 millions $€$ pour l'assurance dépendance (voir tableau 2). Pour l'assurance chômage, la perte est totale car il n'existe pas de versement compensatoire pour les chômeurs. En 2011, l'Agence fédérale pour l'emploi a ainsi vu baisser ses recettes de 1,7 milliard $€$ seulement, alors que le recul atteignait 6,2 milliards $€$ en 2005 . Mais il faut tenir compte de la baisse du taux de cotisation, passé de $6,5 \%$ à $3 \%$ au cours de cette période.

Le moins-perçu fiscal s'élève à 9,5 milliards $€$ en 2011: 8 milliards $€$ sont imputables à l'impôt sur le revenu et 1,5 milliard $€$ à la fiscalité indirecte (TVA). Les ménages ont en effet généralement tendance à limiter leurs dépenses de consommation lorsque leur budget fond pour cause de chômage.

Le montant comme la structure des coûts liés au chômage diffèrent d'un groupe de personnes à l'autre, selon qu'elles ont ou non droit à des prestations et selon le type de revenus de remplacement qu'elles perçoivent le cas échéant.

En 2011, le coût d'un chômeur est ainsi estimé à près de $18900 €$ en moyenne. (voir tableau 4). Ce montant est de $23100 €$ en moyenne pour un bénéficiaire de l'ALG I ( $25 \%$ des chômeurs au total), et de $18700 €$ pour un bénéficiaire de l'ALG II (67 \%). Plus de 8 \% des chômeurs ne perçoivent aucune de ces prestations. Si globalement, le coût budgétaire est nettement plus faible pour ces personnes, il s'élève tout de même à $8600 €$ par tête et par an, principalement en raison de l'absence totale de recettes issues des prélèvements fiscaux et sociaux. Ces personnes assurent en effet leur subsistance pour l'essentiel grâce aux revenus de parents ou concubins soumis à une obligation alimentaire.

Depuis l'année 2005, qui a vu entrer en vigueur l'ensemble des réformes " Hartz », le coût budgétaire global pour ces trois groupes de personnes a connu une baisse tendancielle jusqu'en 2009, avant de remonter légèrement par la suite. Ce recul est dû principalement au fort recul du chômage et, de ce fait, à la baisse du nombre de personnes relevant du régime relativement coûteux de l'assurance chômage (régi par la Chapitre III du Code social allemand, SGB III).

Cela étant, les seuls transferts directs vers les chômeurs ne génèrent que des dépenses comprises entre 4200 et $5000 €$ annuels par tête, soit $27 \%$ au plus du total des coûts. Si on y ajoute les prestations pour les frais de logement et de chauffage, l'allocation logement (Wohngeld) et l'allocation sociale pour les personnes en incapacité de travailler, le montant s'élève alors à $6500 €$, voire $7600 €$, soit $41 \%$ au maximum du total des coûts. Certes, ces montants ne reflètent pas les revenus des personnes touchées par le chômage, car il ne s'agit ici que de transferts moyens calculés sur une base annuelle et se référant aussi bien aux bénéficiaires de l'ALG I ou de l'ALG II qu'aux chômeurs ne percevant aucune de ces prestations. Ils n'en révèlent pas moins que, sous l'angle de vue macroéconomique, le coût du chômage est beaucoup plus important que ne le laisse supposer la considération des seules prestations.

\section{La part des prestations d'assurance baisse, celle des prestations d'assistance augmente}

Les différentes composantes de ce coût ont connu une évolution très différente, voire opposée au fil des années (voir tableau 1). Ainsi, de 2005 à 2011, le fort recul du nombre de chômeurs relevant du périmètre de l'assurance chômage (SGB III) a considérablement allégé la charge financière pesant sur l'Agence fédérale pour l'emploi. Alors que durant cette période, les prestations d'assurance ont été pratiquement divisées par deux, passant de 22 milliards à quelque 12 milliards $€$, les prestations d'assistance sociale n'ont, elles, diminué que d'à peine 6 milliards $€$ en raison d'un nombre de chômeurs relativement stable 
indemnisés au titre du régime d'assistance (SGB II). Ces prestations sociales constituent depuis de nombreuses années le principal poste de dépenses : en 2011, avec 19,1 milliards $€$, elles représentent un peu moins de $34 \%$ du total, avec une tendance à la hausse.

Si durant l'année de récession 2009, mais aussi en 2010, le coût du régime d'assistance (SGB III) avait passagèrement augmenté, pendant que celui du régime d'assistance (SGB II) stagnait, ces coûts ont reculé de respectivement 2 milliards et 1 milliard $€$ en 2011. Les variations de ces différentes composantes reflètent pour l'essentiel l'évolution de la conjoncture, mais aussi la réforme du système de protection sociale en 2005 et la modification qu'elle a entraîné dans la structure du chômage : soit d'un côté, le fort recul du nombre de chômeurs qui relèvent de l'assurance (SGB III) et, de l'autre, la baisse moins rapide et moins prononcée du nombre de ceux relevant de l'assistance (SGB II). Quant au déficit de l'encours fiscal ou des cotisations, il s'est réduit au même rythme que le recul global du chômage.

Ces évolutions ont modifié la structure du coût du chômage. Ainsi, la part des prestations d'assurance a reculé, passant de plus de $25 \%$ en 2005 à un peu moins de $22 \%$ en 2011 , tandis que celle des prestations d'assistance a connu une hausse, passant de $28 \%$ à près de $34 \%$ (voir tableau 1). La part du manque à gagner en termes d'impôts et de cotisations dans le coût global du chômage n'a, elle, que peu varié au fil de ces années.

Le chômage grève les budgets publics de toutes les institutions concernées et à tous les échelons territoriaux. La première institution concernée est l'Agence fédérale pour l'emploi, qui verse les allocations chômage et les cotisations d'assurance maladie, retraite et dépendance afférentes aux chômeurs relevant du SBG III, et qui voit se réduire le volume des cotisations perçues.

Avant les réformes, le Bund assumait le financement de l'aide aux chômeurs de longue durée (Arbeitslosenhilfe) ; depuis la suppression de ce régime en 2005, il finance la nouvelle aide ALG II (" Hartz IV »), une partie des frais de logement et l'allocation sociale pour les personnes en incapacité de travailler (Sozialgeld), tout en percevant moins d'impôts directs et indirects. Les Länder et les communes voient également leurs recettes fiscales diminuer proportionnellement à leur part des impôts communs que sont la TVA, l'impôt sur le revenu et les sociétés (voir REA 102/2011). Les communes, qui finançaient l'aide sociale (Sozialhilfe) jusqu'à la fin 2004, financent depuis aux deux tiers la contribution aux frais de logement et de chauffage versée aux bénéficiaires de l'ALG II, le tiers restant étant à la charge du Bund. Quant à l'allocation logement, elle est financée à parts égales par le Bund et les Länder. La mise en œuvre du « paquet éducation/participation »(Bildungs- und Teihabepaket) pour les enfants et les adolescents issus de ménages bénéficiant de prestations d'assistance (ALG II) incombe certes aux communes, mais c'est le Bund qui en assume le coût. Une part considérable de son coût, estimée à 600 millions $€$ en 2011, est générée par la situation de chômage où se trouvent les membres du foyer soumis à l'obligation alimentaire.

Les caisses d'assurance retraite, maladie et dépendance sont elles aussi concernées, étant donné que les chômeurs bénéficiant des prestations d'assurance et d'assistance versent moins de cotisations que du temps où ils occupaient un emploi ; quant aux chômeurs qui ne peuvent prétendre à aucune prestation, ils ne sont pas soumis à cotisation.

\section{L'Agence fédérale pour l'emploi et le Bund supportent la majeure partie des coûts}

Les calculs réalisés montrent que le coût budgétaire total du chômage est supporté principalement par l'Agence fédérale pour l'emploi et le Bund (voir tableau 2 ) : en 2005-2006, ils en assumaient à eux deux près de $60 \%$; ce n'est que très récemment que ce pourcentage a nettement baissé.
Variations conjoncturelles et réformes...

... ont modifié la structure du coût du chômage

Tous les budgets publics sont concernés 
Fluctuation de la part de l'Agence de Nuremberg

En hausse : part des communes...

... et de l'assurance retraite
Dans le cadre de la réforme du système de protection sociale, la répartition des charges entre eux s'est néanmoins déplacée au profit de l'Agence fédérale pour l'emploi : il y a quatre ans, au plus fort de la croissance, sa part s'était réduite à $26 \%$; durant l'année de récession 2009 en revanche, l'Agence fédérale pour l'emploi a assumé la plus grande part des coûts, avec $33 \%$. La tendance est inverse pour le Bund, qui assumait auparavant une part des coûts supérieure aux $25 \%$ actuels (année 2011). La " contribution d'insertion » (Eingliederungsbeitrag), qui s'appelait « indemnité de fin de droits » (Aussteuerungsbetrag) avant les réformes, et par le biais de laquelle l'Agence fédérale pour l'emploi apporte sa contribution obligatoire à l'aide forfaitaire de base de l'ALG II cofinancée par le Bund, est ici considérée comme une dépense de l'Agence fédérale pour l'emploi, ou comme une recette du Bund, et n'a donc pas d'incidence sur le coût budgétaire global. Sans ces versements, la part supportée par l'Agence de Nuremberg serait plus faible et celle du Bund, plus importante.

Cette évolution des parts respectives au fil du temps résulte pour une large part de la fluctuation, au gré de la conjoncture économique, des dépenses et des recettes de l'Agence fédérale pour l'emploi.

Au fil des années, la part des Länder a légèrement baissé ; elle s'élève à $7 \%$ en 2011. Les communes ont vu en revanche la charge pesant sur elles augmenter (12\% en 2011), notamment en raison du caractère incompressible des prestations sociales relatives au logement, qui leur sont en grande partie imputables.

La part de l'assurance maladie se situe autour des $8 \%$ depuis 2005, la hausse des taux de cotisations l'ayant légèrement fait baisser. La part de l'assurance retraite oscille quant à elle entre $12 \%$ et $16 \%$. Ses charges se sont sensiblement et durablement accrues en 2007 : depuis, les cotisations retraite des chômeurs percevant I'ALG II ont baissé ; leur montant mensuel fixe, qui était de $78 €$, a été ramené à $40 €$ avant d'être supprimé en totalité en 2011.

Le déficit des recettes des assurances retraite, maladie et dépendance s'explique largement par le fait que les cotisations dues par les allocataires de I'ALG I ne sont pas calculées sur la base de la totalité du salaire brut perçu auparavant. Pour les bénéficiaires de l'ALG II, les cotisations sont calculées sur une base forfaitaire à l'image des aides et, par là même, totalement dissociées du revenu précédent ('dé-biographisées'). Pour cette raison, la différence avec les recettes potentielles en cas d'activité des cotisants est considérable. S'ajoute à cela l'absence de cotisations versées pour les chômeurs ne percevant pas de prestations d'assurance ou d'assistance, sauf si ces personnes s'assurent à titre personnel. Quant au 'manque à gagner' de l'Agence fédérale pour l'emploi, il atteint désormais $3 \%$, étant donné que le taux de la cotisation chômage (versée à l'Agence) a été progressivement réduit.

De 2005 à 2011, les charges budgétaires liées au chômage ont connu une évolution très différente d'un organisme à l'autre. Alors que l'Agence fédérale pour l'emploi a vu se réduire de moitié la charge qui lui incombait, les communes et l'assurance retraite n'ont vu la leur reculer d'un sixième seulement.

\section{Coût de la politique active pour l'emploi}

Le bénéfice de la prévention

Les coûts du chômage et ceux de la politique active de l'emploi sont étroitement dépendants, car cette dernière permet d'éviter les situations de chômage, voire d'y mettre un terme, avec toutes les conséquences que cela entraîne en matière de coûts pour l'individu et les budgets publics. II revient alors un rôle clé à toutes ces mesures préventives qui visent le long terme, qu'il s'agisse du chômage partiel, du soutien à la formation professionnelle continue dans l'entreprise ou de l'orientation professionnelle renforcée à l'école. Car si de telles mesures sont appliquées de manière efficiente, elles peuvent contribuer à éviter le chômage et les coûts qui en résultent. 
En 2011, près de 16 milliards $€$ ont été dépensés pour le soutien actif à l'emploi dans le cadre du SGB III et du SGB II. Les indicateurs du tableau 3 mettent en évidence l'importance relative des dépenses allouées et son évolution au cours du temps. Ici, les dépenses consacrées à la politique active pour l'emploi sont rapportées au nombre de chômeurs et au coût du chômage. Les données ainsi calculées révèlent l'important apport budgétaire de la politique pour l'emploi.
Coût :

16 milliards $€$ en 2011

Tableau 3. Dépenses au titre de la politique active pour l'emploi 2001-2011

\begin{tabular}{|c|c|c|c|c|c|c|c|c|c|c|c|}
\hline & 2001 & 2002 & 2003 & 2004 & 2005 & 2006 & 2007 & 2008 & 2009 & 2010 & 2011 \\
\hline Chômeurs inscrits ${ }^{1)}$ (milliers) & 4219 & 4535 & 4830 & 4812 & 4861 & 4487 & 3776 & 3268 & 3423 & 3245 & 2976 \\
\hline $\begin{array}{l}\text { Coût budgétaire total } \\
\text { (hors prestations discrétionnaires }{ }^{2)} \text { ) }\end{array}$ & 76,7 & 83,7 & 91,5 & 92,2 & 87,7 & 82,2 & 67,2 & 55,9 & 59,8 & 60,2 & 56,4 \\
\hline (prestations discrétionnaires $\left.{ }^{2}\right)$ & 1,8 & 2,4 & 3,1 & 3,4 & 3,0 & 3,3 & 3,1 & 3,5 & 2,6 & 1,8 & 1,2 \\
\hline \multicolumn{12}{|c|}{ Dépenses au titre de la politique active pour l'emploi (en milliards $€$ ) } \\
\hline $\begin{array}{l}\text { Mesures facultatives (SGB III) } \\
\text { (Chap. } 2 \text { budget Agence fédérale) }\end{array}$ & 13,9 & 13,5 & 12,1 & 9,1 & 3,6 & 2,5 & 2,5 & 2,9 & 3,6 & 2,9 & 2,3 \\
\hline $\begin{array}{l}\text { Mesures obligatoires (SGB III) } \\
\text { (Chap. } 3 \text { budget Agence fédérale) } \\
\text { dont }\end{array}$ & 8,0 & 8,6 & 8,8 & 9,6 & 10,0 & 8,6 & 7,9 & 7,9 & 13,2 & 12,1 & 8,9 \\
\hline \multirow{2}{*}{$\begin{array}{l}\text { ensemble chômage partiel } \\
\text { ch. partiel conjoncturel (Kug) }\end{array}$} & 0,4 & 0,6 & 0,7 & 0,6 & 0,6 & 0,3 & 0,4 & 0,4 & 5,2 & 4,0 & 1,3 \\
\hline & & & & & 0,4 & 0,2 & 0,1 & 0,1 & 4,6 & 3,1 & 0,7 \\
\hline $\begin{array}{l}\text { Prestations d'insertion SGB II } \\
\text { (Chap. } 1112 \text { budget Bund) }\end{array}$ & & & & & 3,6 & 4,6 & 5,0 & 5,5 & 5,1 & 6,0 & 4,4 \\
\hline Versemt. compensatoire du Bund & 1,9 & 5,6 & 6,2 & 4,2 & 0,4 & & & & & & \\
\hline Dépenses / nbre chômeurs (€/an) & 5191 & 4873 & 4327 & 3886 & 3538 & 3499 & 4078 & 4988 & 6398 & 6471 & 5242 \\
\hline Dépenses/coût global chômage & 0,29 & 0,26 & 0,23 & 0,20 & 0,20 & 0,19 & 0,23 & 0,29 & 0,37 & 0,35 & 0,28 \\
\hline
\end{tabular}

Source des données : calculs de l'IAB. 1) Le nombre de chômeurs considéré inclut, sur la période 2001-2004, le nombre estimé de bénéficiaires de l'aide sociale (ancien régime) capables de travailler et qui, après les réformes entrées en vigueur en 2005, auraient été comptabilisés chômeurs (voir à ce propos IABKurzbericht, $n^{\circ} 14 / 2008$ ). 2) Mesures particulières et mesures transitoires adoptées pour ménager la transition entre les régimes avant/après réforme (SGB II, $\S$ 428 sq. et SGB II $§ 65,4)$.

Les dépenses annuelles relatives à la politique active pour l'emploi ont certes baissé de 1,6 milliard $€$ entre 2005 et 2011 , mais l'effort budgétaire consacré aux mesures de soutien actif au retour en emploi s'est accru : alors que ces dépenses s'élevaient en moyenne à $3500 €$ par chômeur en 2005, elles s'élèvent à $5200 €$ en 2011. Le ratio entre les dépenses consacrées au soutien actif à l'emploi et le coût budgétaire total du chômage a lui aussi subi une hausse durant cette période, passant de 0,2 à 0,28. En 2009, les dépenses exceptionnelles liées au chômage partiel l'ont fait monter provisoirement à 0,37.

Le recul, au cours des années 2001-2004, de l'effort accordé à la politique active pour l'emploi ne s'est pas poursuivi dans la période qui a suivi. II concernait alors les "prestations discrétionnaires » (Ermessensleistungen) du budget de l'Agence fédérale pour l'emploi, c'est-à-dire le titre « mesures d'insertion » (Eingliederungstite/). Celles-ci ont vu leur budget se réduire de près de 5 milliards $€$ entre 2001 et 2004 avec pour conséquence que de moins en moins de chômeurs se voyaient proposer un soutien.

A partir de 2005, avec la réorganisation du financement du chômage, le soutien à l'emploi a regagné en intensité. Les mesures destinées aux bénéficiaires de I'ALG II sont depuis financées par le budget du Bund, alors qu'auparavant, sa contribution au financement de la politique pour l'emploi était essentiellement indirecte, via des subventions versées au budget alors par moments déficitaire de l'Agence fédérale pour l'emploi. Soulignons ici que, après cette nouvelle répartition de la responsabilité budgétaire, l'Agence fédérale pour l'emploi a alimenté le budget du Bund de près de 23 milliards $€$ entre 2005 et 2011, sous la forme de l'indemnité de fin de droits puis de la contribution d'insertion.

La politique active pour l'emploi vise à permettre l'insertion sur le marché du travail du plus grand nombre de personnes bénéficiant d'un soutien, certaines mesures devant permettre à elles seules de réduire le chômage déclaré. Certaines évaluations s'interrogent sur le nombre de participants qui seraient restés au chômage si ces mesures n'existaient pas et qui auraient continué à percevoir allocations chômage ou aide forfaitaire de base (Heyer et al., 2012). A par-

$5200 €$ par chômeur en 2011

Mesures d'insertion : en baisse jusqu'en 2004...

... en hausse depuis 2005

Le bénéfice d'une politique préventive 
Les économies réalisées grâce au chômage partiel tir du moment où ces personnes perçoivent un salaire (sous la forme par exemple des compléments d'insertion), les prestations ne sont plus versées pendant toute la durée d'application de la mesure ; les participants, en leur qualité d'actifs occupés, s'acquittent des impôts et des cotisations sociales. En ce qui concerne les mesures de formation professionnelle continue, la dépense nette se limite aux coûts directement induits par ces mesures, car le versement de l'allocation chômage se poursuit durant la formation continue. Néanmoins, ces mesures de politique pour l'emploi peuvent entraîner des coûts supplémentaires lorsqu'elles ne sont pas utilisées à bon escient et qu'elles empêchent par exemple une personne de reprendre un emploi régulier (risque de lock-in), ou encore lorsque la réinsertion aurait de toute façon eu lieu sans l'aide de ces mesures.

Le coût net est le solde entre le coût total des mesures et le coût supposé_du chômage évité pendant ou après la mise en œuvre des mesures. Selon la mesure adoptée, d'autres effets (positifs ou négatifs) viennent se greffer, qui peuvent avoir eux aussi un impact sur les coûts nets.

L'exemple du chômage partiel conjoncturel (voir REA 90/2009) pendant la crise économique de 2008-2009 montre à quel point les effets budgétaires d'une politique pour l'emploi préventive peuvent être prononcés. Tout semble indiquer en effet que le chômage partiel a préservé l'emploi et ainsi permis de réaliser des économies en matière d'allocations chômage.

En 2009, près de 3 milliards $€$ ont été dépensés pour le chômage partiel conjoncturel (Kug), contre 1,7 milliard en 2010. Les cotisations sociales des personnes au chômage partiel ont été remboursées aux employeurs, respectivement à hauteur de quelque 1,6 et 1,4 milliard $€$.

Avec d'autres formes de flexibilisation du temps de travail, le chômage partiel a amorti en grande partie le choc conjoncturel durant la grande récession de 2009, contribuant ainsi à stabiliser le marché du travail et, par là même, à réduire les coûts du chômage (voir REA 90/2009 et 101/2011). Un calcul type permet d'estimer les économies budgétaires qu'a permis de réaliser le chômage partiel.

L'impact budgétaire net du chômage partiel est fonction notamment du volume des licenciements ainsi évités. En effet, les salariés au chômage partiel sont généralement en droit de percevoir l'ALG I, droit qu'ils mettraient en application s'ils perdaient leur emploi. On peut supposer que le chômage partiel a maintenu de nombreux postes durant la crise, étant donné que les autres mesures de flexibilité avaient toutes été largement épuisées. Dès lors, il n'existait plus guère d'alternative aux licenciements dans ce contexte de forte récession. Quoi qu'il en soit, les études menées à ce titre ne décèlent aucun effet d'aubaine significatif (Scholz/Sprenger/ Bender, 2011).

Quant aux dépenses consacrées au chômage partiel en 2009-2010, elles n'ont pas eu d'impact sur le budget de l'Agence fédérale pour l'emploi (Bach/Spitznagel, 2012). Autrement dit, en l'absence de chômage partiel, pratiquement le même montant aurait été dépensé en allocations chômage supplémentaires. Ainsi, le chômage partiel n'a pas généré de coûts nets durant la crise.

Pour les salariés, les entreprises et pour l'ensemble de l'économie en revanche, le chômage partiel s'est révélé d'une grande utilité. Les salariés ont conservé leur poste, les entreprises les salariés qu'elles avaient formés. Sans cette solution, de nombreuses entreprises auraient pu tout simplement fermer du fait de leur réduction d'activité durant la crise. Dès lors, les pertes en termes de production et d'emploi auraient été encore plus importantes: les recettes auraient plus largement chuté tandis que les dépenses d'indemnités de chômage auraient augmenté. Enfin, le chômage partiel a notamment évité aux entreprises un processus long et coûteux de recrutement après la crise, leur permettant ainsi de sortir rapidement du creux de la vague. Les budgets publics en ont également profité. Avec le recul, le chômage partiel aura été d'un grand bénéfice dans cette récession d'origine exogène.

Bénéfice en termes d'économies de transferts sociaux...

... et de développement du capital humain
Cela dit, considéré à l'instant $\mathrm{T}$, le bilan coûts/avantages (au sens des coûts supposés du chômage) ne reflète pas correctement la justification économique de la politique active pour l'emploi. Car dans la mesure où celle-ci mène à une intégration durable dans le marché de l'emploi, elle permet d'économiser d'éventuels revenus de substitution et d'augmenter dans le même temps les recettes issues des impôts et des cotisations sociales. Toutefois, ces effets réels ne se déploient que dans la mesure où l'intégration ne se fait pas au détriment d'autres personnes; les effets d'aubaine ou d'éviction pourraient amoindrir, voire absorber totalement les économies réalisées.

Les mesures de la politique active pour l'emploi ont d'autres répercussions positives encore : les mesures d'accès à l'emploi et à la formation continue contribuent par exemple à préserver, voire à développer le capital économique, mais aussi le capital humain. Or de tels effets réels ne se manifestent que progressivement, et il est particulièrement difficile de quantifier l'impact des mesures d'insertion sur la réduction ou la prévention des coûts psycho-sociaux et sanitaires du chômage (Allmendinger et al., 2012). Autrement dit, il existe en- 
core un besoin considérable de recherche sur la « valeur ajoutée de la politique de l'emploi » (Schmid, 2007).

Tableau 4. Coût budgétaire global du chômage en Allemagne 2005-2011

\begin{tabular}{|c|c|c|c|c|c|c|c|c|}
\hline & & 2005 & 2006 & 2007 & 2008 & 2009 & 2010 & 2011 \\
\hline Chômeurs inscrits & 1000 & 4861 & 4487 & 3776 & 3268 & 3423 & 3245 & 2976 \\
\hline \multicolumn{9}{|l|}{ dont } \\
\hline allocataires ALG I & $\%$ & 29,2 & 24,8 & 20,7 & 18,3 & 26,6 & 26,6 & 24,6 \\
\hline bénéficiaires ALG II & $\%$ & 54,2 & 59,8 & 64,5 & 66,2 & 62,6 & 64,2 & 67,3 \\
\hline sans prestations & $\%$ & 16,6 & 15,4 & 14,8 & 15,5 & 10,8 & 9,2 & 8,1 \\
\hline Coût par chômeur & $1000 €$ & 18,0 & 18,3 & 17,8 & 17,1 & 17,5 & 18,5 & 18,9 \\
\hline allocataires ALG I & $1000 €$ & 22,9 & 23,4 & 22,6 & 21,5 & 21,1 & 22,7 & 23,1 \\
\hline bénéficiaires ALG II & $1000 €$ & 18,0 & 18,5 & 18,2 & 17,8 & 17,5 & 18,2 & 18,7 \\
\hline sans prestations & $1000 €$ & 9,6 & 9,6 & 9,2 & 8,8 & 8,4 & 8,8 & 8,6 \\
\hline $\begin{array}{l}\text { Coût budgétaire global } \\
\text { dont }\end{array}$ & milliards $€$ & 87,7 & 82,2 & 67,2 & 55,9 & 59,8 & 60,2 & 56,4 \\
\hline Prestations d'assurance ${ }^{1 \text { ) }}$ & milliards $€$ & 22,2 & 17,6 & 12,3 & 9,0 & 13,8 & 14,0 & 12,1 \\
\hline ALG I (chômeurs seuls) & milliards $€$ & 13,1 & 10,4 & 7,2 & 5,3 & 8,0 & 8,2 & 7,1 \\
\hline cotisations maladie & milliards $€$ & 3,6 & 2,8 & 2,0 & 1,5 & 2,3 & 2,3 & 2,1 \\
\hline cotisations retraite & milliards $€$ & 5,1 & 4,0 & 2,8 & 2,1 & 3,1 & 3,2 & 2,8 \\
\hline cotisations dépendance & milliards $€$ & 0,4 & 0,3 & 0,2 & 0,2 & 0,3 & 0,3 & 0,3 \\
\hline Prestations sociales (assistance) ${ }^{2)}$ & milliards $€$ & 24,6 & 25,7 & 22,7 & 20,6 & 20,6 & 20,2 & 19,1 \\
\hline ALG II (chômeurs seuls) & milliards $€$ & 9,3 & 10,0 & 9,3 & 8,4 & 8,3 & 8,1 & 7,7 \\
\hline cotisations maladie & milliards $€$ & 2,9 & 2,8 & 2,7 & 2,5 & 2,5 & 2,4 & 2,5 \\
\hline cotisations retraite & milliards $€$ & 2,7 & 2,8 & 1,2 & 1,0 & 1,0 & 1,0 & 0,0 \\
\hline cotisations dépendance & milliards $€$ & 0,4 & 0,4 & 0,3 & 0,3 & 0,4 & 0,4 & 0,4 \\
\hline Aide complémentaire (§24 SGB II) & milliards $€$ & 0,5 & 0,3 & 0,2 & 0,1 & 0,1 & 0,1 & 0,0 \\
\hline Aide complémentaire à l'ALG I & milliards $€$ & 0,3 & 0,3 & 0,2 & 0,2 & 0,3 & 0,2 & 0,2 \\
\hline Sozialgeld (enfants, conjoint du chômeur) & milliards $€$ & 0,4 & 0,5 & 0,4 & 0,4 & 0,4 & 0,4 & 0,3 \\
\hline aide au logement & milliards $€$ & 0,1 & 0,1 & 0,1 & 0,0 & 0,1 & 0,1 & 0,1 \\
\hline Prise en charge frais logement/chauffage & milliards $€$ & 8,0 & 8,6 & 8,3 & 7,6 & 7,5 & 7,4 & 7,3 \\
\hline Teilhabe/Bildungspaket (aide scolaire) & milliards $€$ & & & & & & & 0,6 \\
\hline \multirow{3}{*}{$\begin{array}{l}\text { Déficit encours fiscal } \\
\text { impôt sur le revenu } \\
\text { impôts indirects }\end{array}$} & milliards $€$ & 16,2 & 15,0 & 12,1 & 9,8 & 10,0 & 10,3 & 9,5 \\
\hline & milliards $€$ & 13,1 & 12,3 & 10,0 & 8,1 & 8,5 & 8,7 & 8,0 \\
\hline & milliards $€$ & 3,1 & 2,8 & 2,1 & 1,7 & 1,5 & 1,6 & 1,5 \\
\hline \multirow{5}{*}{$\begin{array}{l}\text { Déficit encours cotisations } \\
\text { assurance maladie (solde) } \\
\text { assurance retraite (solde) } \\
\text { assurance dépendance (solde) } \\
\text { Agence fédérale pour l'emploi }\end{array}$} & milliards $€$ & 24,7 & 23,8 & 20,1 & 16,5 & 15,4 & 15,7 & 15,6 \\
\hline & milliards $€$ & 7,0 & 6,8 & 6,0 & 4,9 & 4,6 & 4,7 & 4,3 \\
\hline & milliards $€$ & 10,7 & 10,4 & 10,5 & 8,9 & 8,4 & 8,5 & 9,1 \\
\hline & milliards $€$ & 0,8 & 0,8 & 0,7 & 0,7 & 0,6 & 0,7 & 0,6 \\
\hline & milliards $€$ & 6,2 & 5,8 & 3,1 & 2,0 & 1,8 & 1,8 & 1,7 \\
\hline \multicolumn{9}{|c|}{ En outre : indemnité de fin de droits, puis à partir de 2008 , contribution d'insertion } \\
\hline Surcroît de dépenses BA & milliards $€$ & 3,7 & 2,5 & 1,4 & 3,3 & 3,9 & 4,4 & 4,0 \\
\hline Surplus de recettes Bund & milliards $€$ & 3,7 & 2,5 & 1,4 & 3,3 & 3,9 & 4,4 & 4,0 \\
\hline Agence fédérale pour l'emploi & milliards $€$ & 32,1 & 25,9 & 16,7 & 14,3 & 19,4 & 20,2 & 17,8 \\
\hline Bund & milliards $€$ & 22,4 & 23,8 & 21,2 & 16,4 & 15,7 & 14,7 & 13,9 \\
\hline Länder & milliards $€$ & 6,7 & 6,2 & 5,0 & 4,0 & 4,2 & 4,3 & 3,9 \\
\hline Communes & milliards $€$ & 8,0 & 8,3 & 7,2 & 6,6 & 6,9 & 7,0 & 6,7 \\
\hline Assurance maladie & milliards $€$ & 7,0 & 6,8 & 6,0 & 4,9 & 4,6 & 4,7 & 4,3 \\
\hline Assurance retraite & milliards $€$ & 10,7 & 10,4 & 10,5 & 8,9 & 8,4 & 8,5 & 9,1 \\
\hline Assurance dépendance & milliards $€$ & 0,8 & 0,8 & 0,7 & 0,7 & 0,6 & 0,7 & 0,6 \\
\hline \multicolumn{9}{|c|}{$P M$ : Surcroît de dépenses et déficit de recettes pour les bénéficiaires de prestations qui ne sont pas demandeurs d'emploi ${ }^{3)}$} \\
\hline Bénéficiaires de prestations & 1000 & 541 & 627 & 646 & 738 & 678 & 674 & 572 \\
\hline Coût & milliards $€$ & 11,3 & 13,2 & 13,1 & 14,4 & 12,8 & 13,1 & 11,2 \\
\hline
\end{tabular}

Source des données : calculs de l'IAB. 1) Hors allocataires handicapés, bénéficiant de mesures facultatives ou transitoires (§ 125, 126 et 428 SGB III) ou participant à des mesures de requalification, et hors placement confié à des tiers. 2) Hors bénéficiaires de mesures transitoires (\$ 65, 4 SGB II) ou participant à des mesures de requalification, et hors placement confié à des tiers. 3) Compte tenu des personnes citées aux notes 1) et 2).

LES RÉSULTATS DE CETTE ANALYSE MONTRENT CLAIREMENT que le chômage induit des charges budgétaires à différents niveaux institutionnels, aussi bien dans les finances publiques et que dans les comptes sociaux. Bien souvent, leur importance ne frappe pas à première vue. Et pourtant, elles grèvent lourdement les budgets publics. Les réformes structurelles des prestations et l'augmentation de la part de la fiscalité dans le financement de ces dernières n'y ont pas changé grand-chose, bien qu'elles aient considérablement contribué à soulager le facteur travail des charges qui pesaient sur lui. Malgré cela, la problématique du 
" sous-emploi » est rarement considérée dans une perspective globale, tenant compte des coûts induits pour tous les budgets concernés - en Allemagne comme ailleurs. Le coût élevé du chômage, sa répartition et plus particulièrement son origine devraient pourtant davantage être pris en compte dans les débats portant sur les mesures de réduction du chômage.

Par-delà le coût budgétaire, le chômage peut générer d'autres types de coûts (résultant notamment du chômage de longue durée), qui sont toutefois difficiles à quantifier. Ces incidences négatives n'apparaissent qu'après un examen approfondi. Ainsi, le chômage peut mener à un déclassement professionnel de la personne concernée ou à la dévalorisation progressive de son capital humain. Les nuisances psycho-sociales et sanitaires induites par le chômage ont dès lors elles aussi une importance considérable. Les liens possibles entre chômage, radicalisme et criminalité, ainsi que les conséquences sur le long terme des problèmes de socialisation professionnelle (des jeunes notamment), sont autant d'autres effets dont il convient de se préoccuper. Le chômage peut réduire la motivation au travail et l'engagement sociétal des personnes concernées. Tout cela est très onéreux, particulièrement sur le long terme. II convient donc de ne pas considérer seulement le chômage sous l'angle de son coût économique et budgétaire, mais aussi et avant tout dans la perspective de la cohésion et de la préservation du lien social.

(Traduction : I. Bourgeois et S. Hazouard)

\section{Indications bibliographiques}

-AChATZ J., TRAPPMANN M., " Wege aus der Grundsicherung », IAB-Kurzbericht, n² 28, 2009

- Allmendinger J., LudWig-Meyerhofer W., Spitznagel E., « Arbeitslosigkeit », in Albrecht G., Groenemeyer A. (Hrsg.), Handbuch soziale Probleme, 2. überarbeitete Auflage, Vol. 1, 2012

-BACH H.-U., SpItznagel E., "Kosten der Arbeitslosigkeit. Druck auf öffentliche Budgets lässt nach », IAB-Kurzbericht, 8/2012

-BACH H.-U., SPITZNAGel E., « Staatsfinanzen : Kosten der Arbeitslosigkeit sind gesunken », IAB-Kurzbericht, $n^{\circ} 14,2008$

-Dietz M., DitTRich L. M., Stops M., WalWei U., «Beschäftigungssicherung durch Arbeitskräftehorten », Sozialer Fortschritt, vol. 10, 2011

- Fuchs J., hutter C., Klinger S., Spitznagel E., Weber E., Zapf I., Zika G., «Arbeitsmarktprognose 2012 : Der Aufwärtstrend flacht ab », IAB-Kurzbericht, n 3, 2012

- HeYer G., Koch S., Stephan G., Wolff J., «Evaluation der aktiven Arbeitsmarktpolitik: Ein Sachstandsbericht für die Instrumentenreform 2011 », Journal for Labour Market Research, Vol. 45, n 1, 2012

-HoRn G., Tober S., « Wie stark kann die deutsche Wirtschaft wachsen ? Zu den Irrungen und Wirrungen der Potenzialrechnung », IMK-Report Nr. 17, 2007

-KolLeR, L., «Lohnmobilität alleinstehender SGB-II-Leistungsempfänger », IAB-Discussion Paper, $n^{\circ} 5$, 2011

-KOLLER L., RUDOLPH H., " Arbeitsaufnahmen von SGB-II-Leistungsempfängern : Viele Jobs von kurzer Dauer », IAB-Kurzbericht , n¹4, 2011

-PRotsch P., «Wachsende Unsicherheiten: Arbeitslosigkeit und Einkommensverlust bei Wiederbeschäftigung », WZB-Discussionpaper SP I 2008-506

-Schmid G., "Der Mehrwert der Arbeitsmarktpolitik », Contribution lors de la conférence de la Deutsche Vereinigung für Sozialwissenschaftliche Arbeitsmarktforschung (SAMF) en décembre 2007 au Wissenschaftszentrum Berlin für Sozialforschung (WZB), 2007

-Scholz T., SpRenger C., Bender S. "Kurzarbeit in Nürnberg : Beruflicher Zwischenstopp oder Abstellgleis? ", IAB-Kurzbericht, $\mathrm{n}^{\circ}$ 15, 2011. 